\title{
Research on Optical Energy Storage System Based on Rule Logic Control
}

\author{
Lu Yan ${ }^{1, *}$, Rui Xu ${ }^{1}$, and Xin Zhang ${ }^{1}$ \\ ${ }^{1}$ School of Electrical Engineering, Shanghai Dianji University, 201306 Shanghai, China
}

\begin{abstract}
Photovoltaic energy storage system is composed of photovoltaic power generation, energy storage battery, load and power grid, it has researched on different modes of bi-directional DC/AC converter, taking into account a variety of photovoltaic output fluctuations, energy storage battery charging and discharging management, load increasing and decreasing, etc. Aiming at the energy management strategy, using DC bus voltage fluctuation as the basis of energy layer mobilization, the power balance control based on regular control is proposed. Compared with the DC bus voltage level energy management, the power tracking accuracy is further improved and energy loss is reduced. Finally, through MATLAB/Simulink being in off/connected model and the charging/discharging model, the effectiveness of the energy control strategy was verified separately by simulation. The simulation results show that: the proposed control strategy can improve the power tracking accuracy and reduce the energy loss of the system; the proposed strategy guarantees the bidirectional exchange of system energy and maintains the power balance in the network, the DC bus voltage is stable, and the energy storage battery achieves smooth power output and peak load shifting.
\end{abstract}

\section{Introduction}

In the field of new energy power generation, such as photovoltaic power generation and wind power generation, there are volatility, intermittentness and randomness. These unavoidable factors will cause output power fluctuations and bring non-negligible negative effects on the grid and load. The integrated application of energy and energy storage devices can help improve the local consumption of renewable energy and solve the power fluctuations in the grid caused by intermittent energy output [1-3]. How to solve the time and space mismatch between production capacity and energy use through energy storage, and realize the efficient use of large-scale renewable energy is the focus of the energy Internet research. At the same time, because it contains a variety of energy flow conditions, how to achieve different units Inter-energy management and coordinated control are also a major research focus. In response to this hot spot, a lot of research has been done in related literature [4-6].

Aiming at the problem that photovoltaic power generation is affected by external environmental factors such as light intensity and temperature, the output power fluctuates, and the stability is affected when it is connected to the grid. An energy storage battery is designed as an energy storage device to achieve complementary solar storage systems. The energy of each part is the flow takes the DC bus as the connection point. It is proposed to use the DC bus voltage fluctuation as the basis for energy layer transfer, and the power balance control based on rule logic control as the corresponding energy management control strategy to realize the load, energy storage battery, photovoltaic power generation and power grid. Energy flow between. The charging and discharging effect of the energy storage battery realizes smooth photovoltaic output power fluctuations, so that the power of photovoltaic power input to the grid remains stable for a certain period of time, realizes the function of peak shaving and valley filling, and reduces the impact of intermittent power output fluctuations on power quality.

\section{Optical storage system topology}

The composition diagram of the photovoltaic energy storage system is shown in Figure 1. The entire system is output by photovoltaic modules through maximum power tracking (MPPT) to ensure the maximum utilization of photovoltaic energy. The energy storage converter (PCS) is used to realize the two-way exchange of energy with the grid, and the energy storage battery is used to solve the fluctuation of photovoltaic output. The energy required by the load is first met by photovoltaic output, and the shortfall is provided by the grid or energy storage batteries. Energy storage batteries mainly ensure that the energy flow within the grid is in a balanced state. Finally, a complementary system model for optical storage is established to achieve smooth output. 


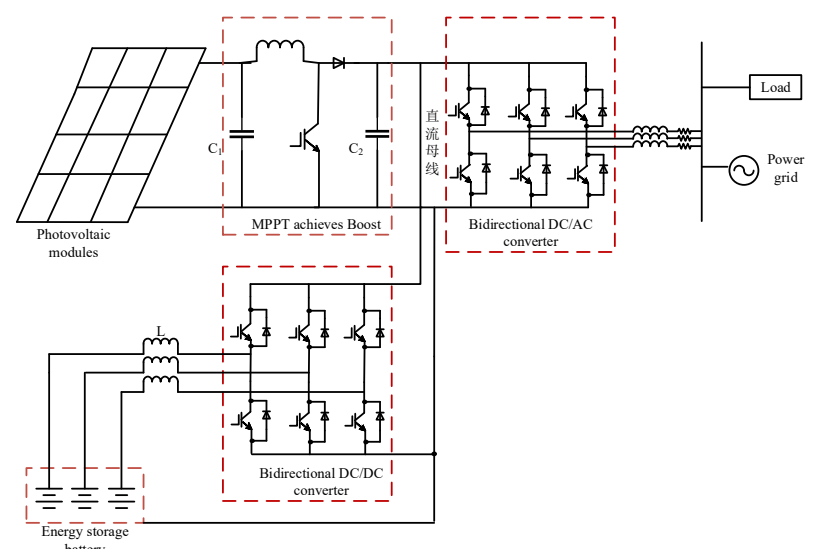

Fig1. Photovoltaic energy storage system composition diagram

\section{Optical storage system rules control operation mode}

\subsection{System energy management solution}

During the operation of the entire optical storage system, its control principle is shown in Figure 2. Among them, the DC bus voltage calculation module calculates the realtime value of the DC bus voltage according to the output voltage of different units; the working state selection module compares the calculated value of the DC bus with the set threshold to determine the working state of the energy storage converter; the power tracking calculation module is based on At this moment, the photovoltaic output and the power required by the load calculate the charge and discharge power of the energy storage battery; the mode selection module performs power distribution according to the working status and the power calculation result, and controls the bidirectional DC/DC converter for power throughput.

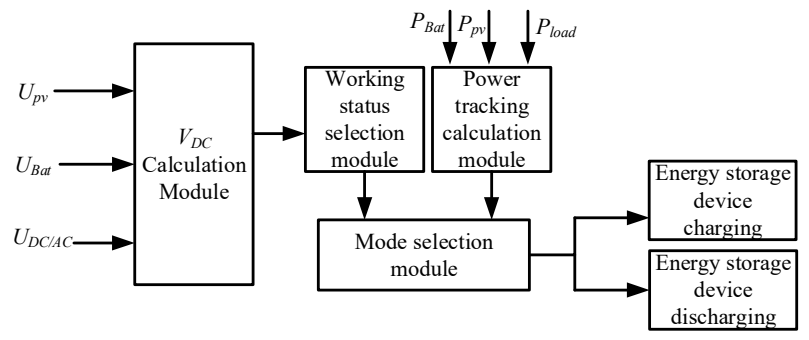

Fig2. System composition diagram of system energy management

In the figure: $\mathrm{U}_{\mathrm{PV}}, \mathrm{U}_{\mathrm{Bat}}, \mathrm{U}_{\mathrm{DC} / \mathrm{AC}}$ are the photovoltaic output voltage, energy storage battery output voltage, and grid-side converter transmission power converted to the DC side voltage respectively. $\mathrm{P}_{\mathrm{PV}}, \mathrm{P}_{\mathrm{Bat}}$, and $\mathrm{P}_{\text {load }}$ are the output of the photovoltaic module, the output power of the energy storage battery, and the power required by the load, respectively.

\subsection{Regular control mode and energy flow state}

The flow of power in the network is ultimately directly reflected in the increase and decrease of the DC bus voltage. Maintaining the power balance in the network is equivalent to stabilizing the DC bus voltage. Therefore, the system operating mode switching is based on the DC bus voltage. In order to ensure the optimization of mode selection and improve the operating efficiency of the proposed smooth control strategy, a rule-based control strategy is designed according to mode selection and power balance [7]. Its core content is: the collected data is allocated according to the established principles for rule configuration, and when the corresponding conditions are met, it enters the corresponding mode to work [8]. The research of rule control is based on the DC bus voltage and power characteristics in the network under different working conditions. The specific control process is shown in Figure 3. $\mathrm{U}_{\mathrm{dc}}$ is the measured voltage value, $\mathrm{U}_{\mathrm{H} 2}$ represents the DC bus voltage is higher due to power surplus on the DC side, close to $1.1 \mathrm{U}_{\mathrm{dc}}{ }^{*}$; $\mathrm{UH} 1$ represents the DC bus has a slightly higher fluctuation than the normal voltage, approximately $1.05 \mathrm{U}_{\mathrm{dc}}{ }^{*}$; $\mathrm{UL} 1$ represents the DC bus is normal Slightly lower fluctuations, approximately $0.95 \mathrm{U}_{\mathrm{dc}}{ }^{*} ; \mathrm{U}_{\mathrm{L} 2}$ means that the $\mathrm{DC}$ bus is low due to power shortage on the DC side, approximately 0.9 $\mathrm{U}_{\mathrm{dc}}{ }^{*}[9]$. The whole system is based on the DC bus voltage adjustment to realize power balance control to ensure voltage balance and power balance within the network.

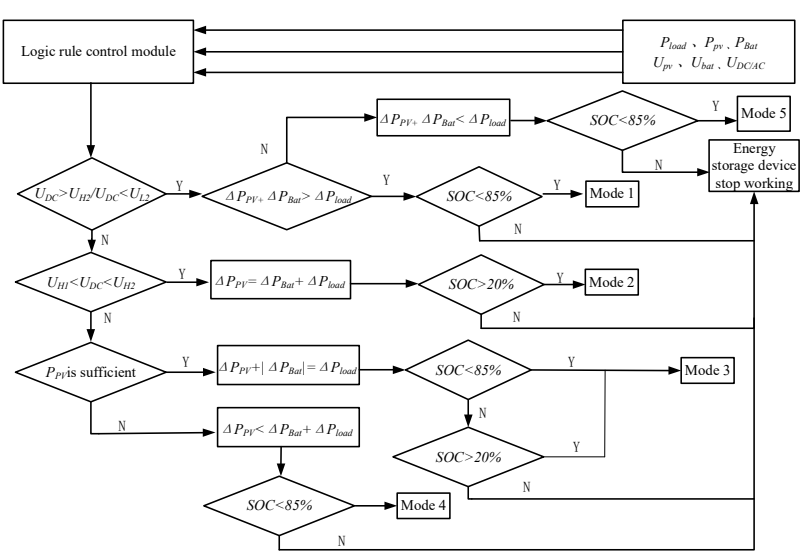

Fig3. Regular control flow chart

\section{Simulation and experiment}

\subsection{Comparative analysis of energy management strategies}

Using different energy management strategies to track the actual load demand and photovoltaic output, the results are shown in Figure 6 and Figure 7. The results show that the energy management strategy based on rule logic control reduces the tracking loss of photovoltaic output and load forecasting, and improves the power tracking accuracy. 


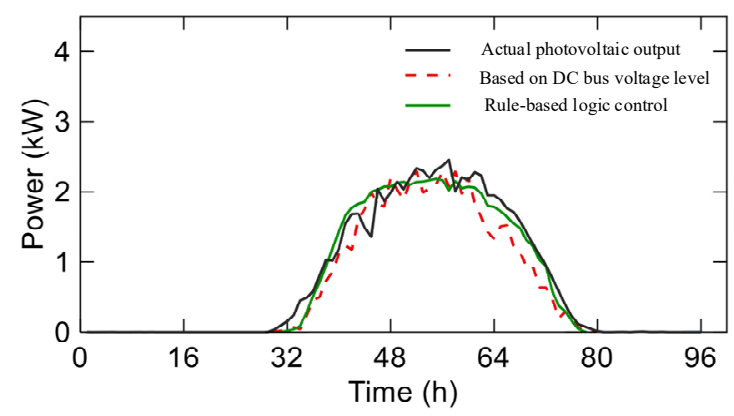

Fig4. Load demand tracking

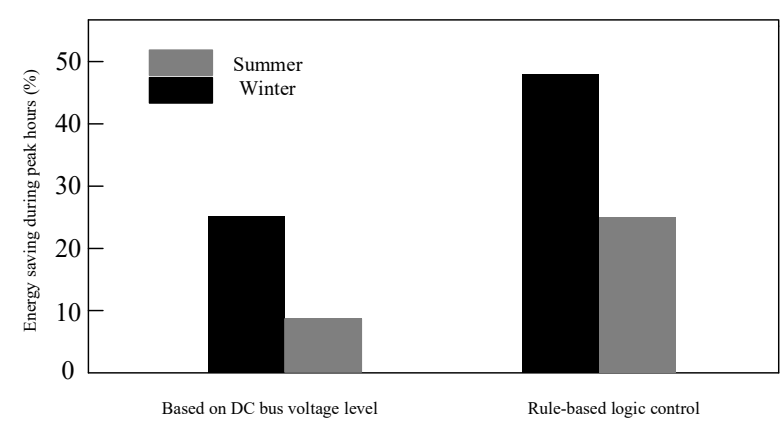

Fig5. Save energy during peak hours(\%)

\subsection{Mode switching simulation}

\subsubsection{Grid-connected state mode switching simulation}

The initial operating light intensity of the system is $600 \mathrm{~W} / \mathrm{m}^{2}$. The lack of power on the DC side causes a large drop in the DC side bus voltage, and the energy storage battery power supply energy is insufficient. The system working mode is 5, the PCS works in the rectifying state, and the $\mathrm{AC}$ side delivers energy to charge the battery. The light intensity rises to $1000 \mathrm{~W} / \mathrm{m}^{2}$ by $0.8 \mathrm{~s}$, the photovoltaic output is increased from the original $196 \mathrm{~kW}$ to $498.9 \mathrm{~kW}$, the system working mode is switched from 5 to 1 , the photovoltaic output is sufficient and the energy storage battery still has remaining power transmitted to the grid after working at the upper limit of charge In the whole process, the DC bus voltage is basically stable at about $715 \mathrm{~V}$.

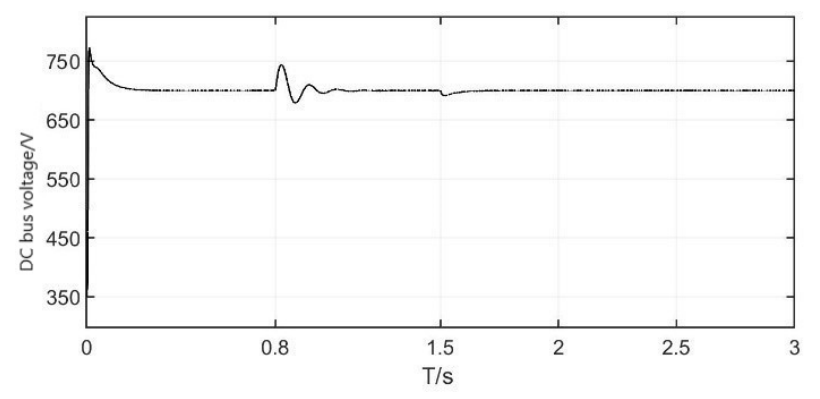

Fig6. DC voltage bus

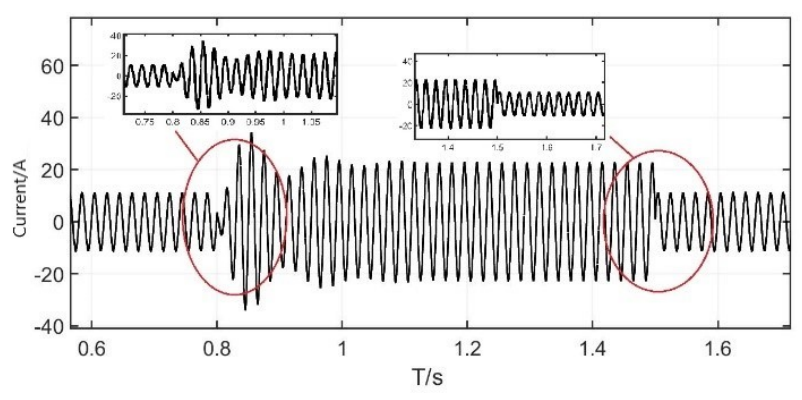

Fig7. Rectifier and inverter mode switching

\subsubsection{Simulation analysis of off-grid state mode switching}

When operating off-grid, photovoltaic output and energy storage battery output jointly maintain power balance while ensuring that photovoltaics are used to the maximum.

When the photovoltaic output is $498.9 \mathrm{~kW}$ in $0-0.8 \mathrm{~s}$, when the operation reaches $0.8 \mathrm{~s}$, due to the change of the external environment, the light intensity suddenly drops from $1000 \mathrm{~W} / \mathrm{m}^{2}$ to $600 \mathrm{~W} / \mathrm{m}^{2}$ and the output power drops to $196 \mathrm{~kW}$. Figure 8 shows the power required by the load, which is $350 \mathrm{~kW}$ for $0-1.5 \mathrm{~s}$ and $450 \mathrm{~kW}$ for $1.5 \mathrm{~s}-3 \mathrm{~s}$.

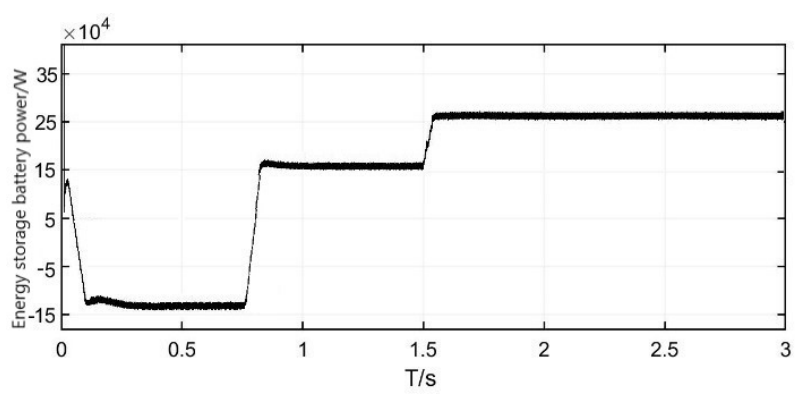

Fig8. Energy storage battery power

\section{Conclusion}

This article aims to stabilize the DC bus voltage and accurately track the actual demand of photovoltaic output and load by the energy storage battery to achieve peak shaving and valley filling. The power balance control based on rule logic control is designed to achieve 5 modes of optical storage system operation, and then the two-way DC/ Control strategies for DC converters and energy storage converters.

The simulation results show that the energy management strategy based on rule-based logic control proposed in this paper corresponds the working mode of the energy storage converter and the charging and discharging state of the energy storage battery to the specific working conditions, and solves the problem that may be caused by the fluctuation of photovoltaic output when the external environment changes. System power imbalance problem, and stabilize the DC bus voltage, use energy storage battery to achieve smooth output power, and achieve the goal of peak clipping and valley filling. At the same time, it achieves accurate tracking of photovoltaic output and load demand, and improves power tracking accuracy, which proves the effectiveness 
of this strategy in reducing system energy loss and improving energy utilization.

\section{References}

1. LC Wang, YP Gao. Electrical Measurement \& Instrumentation, [J].52(14): 49-54.(2015).

2. M Ding, JX Shi, PP Han. China Electric Power[J/OL]: 1-7[2020-04-24].

3. Kai Sun,Li Zhang,Yan Xing. IEEE Transactions on Power Electronics. 2011

4. Yao Bo, Fan Yanfang. Renewable Energy[J]. 2017, 35(02): 232-239.

5. Wang Yi, Zhang Lirong, Li Heming, Liu Junpeng. Proceedings of the Chinese Society of Electrical Engineering[J]. 2013, 33(04): 16-24+4.

6. Xue Zhang, Pei Wei, Deng Wei, Qu Hui, Shen Ziqi, Zhao Zhenxing. Proceedings of the Chinese Society of Electrical Engineering, 2014, 34(31):5553-5562.

7. BANVAIT H,ANWAR S. 2009 American control conference. 2009

8. Zhendong Zhang. Hubei University of Technology, 2018.

9. Maoliang Hu. University of Electronic Science and Technology of China, 2014. 